\title{
A Nonlinear Goal Programming Model for University Admission Capacity Planning with Modified Differential Evolution Algorithm
}

\author{
Said Ali El-Quliti, ${ }^{1}$ Abdul Hamid Mohamed Ragab, ${ }^{2}$ Reda Abdelaal, ${ }^{1}$ Ali Wagdy Mohamed, ${ }^{3}$ \\ Abdulfattah Suliman Mashat, ${ }^{4}$ Amin Yousef Noaman, ${ }^{5}$ and Abdulrahman Helal Altalhi ${ }^{6}$ \\ ${ }^{1}$ Industrial Engineering Department, Faculty of Engineering, King Abdulaziz University, P.O. Box 80203, Jeddah 21589, Saudi Arabia \\ ${ }^{2}$ Information Systems Department, Faculty of Computing and Information Technology, King Abdulaziz University, \\ P.O. Box 80203, Jeddah 21589, Saudi Arabia \\ ${ }^{3}$ Statistics Department, Faculty of Sciences, University of Jeddah, Jeddah 21589, Saudi Arabia \\ ${ }^{4}$ Deanship of Admission \& Registration, King Abdulaziz University, P.O. Box 80203, Jeddah 21589, Saudi Arabia \\ ${ }^{5}$ Computer Science Department, Faculty of Computing and Information Technology, King Abdulaziz University, \\ P.O. Box 80203, Jeddah 21589, Saudi Arabia \\ ${ }^{6}$ Information Technology Department, Faculty of Computing and Information Technology, King Abdulaziz University, \\ P.O. Box 80203, Jeddah 21589, Saudi Arabia
}

Correspondence should be addressed to Ali Wagdy Mohamed; awelnaggar@kau.edu.sa

Received 1 April 2015; Revised 16 May 2015; Accepted 19 May 2015

Academic Editor: Erik Cuevas

Copyright (C) 2015 Said Ali El-Quliti et al. This is an open access article distributed under the Creative Commons Attribution License, which permits unrestricted use, distribution, and reproduction in any medium, provided the original work is properly cited.

\begin{abstract}
This paper proposes a nonlinear Goal Programming Model (GPM) for solving the problem of admission capacity planning in academic universities. Many factors of university admission capacity planning have been taken into consideration among which are number of admitted students in the past years, total population in the country, number of graduates from secondary schools, desired ratios of specific specialties, faculty-to-students ratio, and the past number of graduates. The proposed model is general and has been tested at King Abdulaziz University (KAU) in the Kingdom of Saudi Arabia, where the work aims to achieve the key objectives of a five-year development plan in addition to a 25-year future plan (AAFAQ) for universities education in the Kingdom. Based on the results of this test, the proposed GPM with a modified differential evolution algorithm has approved an ability to solve general admission capacity planning problem in terms of high quality, rapid convergence speed, efficiency, and robustness.
\end{abstract}

\section{Introduction}

Higher education is one of the most important assets for the development of human resources and strategic development. In fact, through higher education programs, job market needs are satisfied in terms of labor and expertise required by national development plans. Higher education throughout the world is witnessing major changes, transformations, and challenges prompted by the developments required by current technology and modernization. On the other side, higher education sector in the Kingdom of Saudi Arabia has observed great leaps during the years 2009-2013. Massive development plans that covered all aspects of the educational process were successfully applied over all regions of the Kingdom and in various provinces. There were 15 universities in 2004 , the beginning of 8 th development plan; they became 32 universities, 8 of which were private by the end of this plan in 2008 [1]. This increase in the number of universities was due to the increase in the students graduated from the secondary schools which correspondingly created a required expansion in the capacity of the existing universities. Enrollment management has been defined as any institutional attempt to influence the number, mix, and quality of students through recruitment and retention strategies [2, 3]. Many external 
factors are influencing the process, mainly the number of high school graduates, the percentage of graduates deciding to attend college, higher education specialties, student-tofaculty ratio, number of populations in the higher education age, government policies, employment opportunities, and available budget and resources. Many attempts have been made to mathematically model a variety of problems in admission capacity planning that were applied to different problems $[4,5]$. One of the most powerful tools is to use Goal Programming (GP) since many problem goals are conflicting and someone can only satisfy a subset of these goals to some extent according to the priority and weights given to specific goals, as explained in this paper.

It is known that GP is an extension of linear programming involving an objective function with multiple objectives [6]. The traditional GP model can be easily solved by simplex method or by using computerized software as Microsoft Excel Solver add-on and the LINGO package [7]. However, it should be noted that there are many other types of GP models that may include large-scale and nonlinear relations, such models with large number of integer variables which add a computational challenge and extra level of difficulty for solving using classical programming techniques. Consequently, using metaheuristic techniques as a substitute for traditional programming methods in order to solve hard GP problems is an open research area $[8,9]$. Thus, due to the complexity of the proposed model, an enhanced constrained differential evolution algorithm is developed to solve the proposed nonlinear integer GP model.

The paper is organized as follows: Section 2 handles the literature review for the problem under study. Section 3 explains the key objectives of the five-year development plan and the 25-year future plan (AAFAQ) for universities education in Saudi Arabia. Section 4 introduces the GPM model for the admission problem. Section 5 shows how the proposed mathematical GPM developed to adapt KAU admission, as a case study for different scenarios. A proposed differential evolution approach and the problem solution are explained in Section 6. The conclusions and points for future researches are summarized in Section 7.

\section{Literature Review}

GP is a powerful and appropriate technique for optimizing university admission planning. Applications of GP were used in modeling university planning appearing in early 1970s by Lee and Clayton [10]. In 1974 Lee and Moore [11] formulated a GP model suitable for United States institution's administrative policies. The goal was to develop a GP model for formulation of admission policy for newly entering students.

In 1981, Kendall and Luebbe [12] developed a GP model to manage recruitment activities in the small four-year Concordia College in Nebraska. These activities included budget, time, manpower, and marketing strategies. The goal was to enable recruiters to meet enrollments while managing recruiting resources and activities in order to remain within the recruiting budget. They concentrated on university financial related problems for private colleges.
In 2009, Khan [13] used a product mix model of linear programming for university's optimal enrollment management. The aim was to obtain the best tuition contribution to the campus using the best student mix and optimal use of those constraints that impact student enrollment every semester.

In 2001, Gottlieb [14] had discussed admissions offices at selective colleges. The aim was to balance a number of conflicting issues in deciding which applicants to admit. He discussed the variables, constraints, and issues involved in modeling the admissions problem using an integer programming model. He used certain statistics, such as applicants' acceptance rate, to rate colleges by organizations such as U.S. News and World Report.

In 2010, Sugrue [15] described the application of linear programming as a decision tool in merit based financial aid decisions at a medium size private university. The objective of the model was to maximize the quality of the incoming class as measured by average combined SAT scores.

In 2012, Mashat et al. [16] proposed an effective decision support system based on an absorbing Markov model, which is used for helping decision makers at King Abdulaziz University in controlling student's flow transition enrollment.

In 2013, Kassa [17] used a linear programming approach for placement of applicants to study programs developed and implemented at the College of Business and Economics, Bahir Dar University in Ethiopia. The approach is estimated to significantly streamline the placement decision process at the college by reducing required man-hour as well as the time it takes to announce placement decisions.

Recently, in 2014, Ragab et al. [18] proposed an efficient classification algorithm for students' college enrollment approval using data mining. And in [19], they presented a new college admission system using hybrid recommender based on data mining techniques and knowledge discovery rules for tackling college admissions prediction problems.

From the above literature review, it is clear to conclude that these studies were limited to theoretical possibilities and one-time applications. One could draw a conclusion that each approach being carried out has appropriate and individual differences in colleges which may not allow generalizing of their results. Therefore, there is a definite need for a planning model which considers the timeliness of admission and enrollment activities as well as their general effectiveness, as proposed in this working paper.

\section{Development Plan and AAFAQ Project Objectives}

The 9th national development plan 2010-2014, of the Kingdom of Saudi Arabia (KSA), came at a crucial distinctive point in the history of the development of the Kingdom. Subsequent to the remarkable comprehensive achievements and the quantum leap accomplished by the Kingdom since the inception of its five-year plans, nearly four decades ago, the 9th five-year plan came at a time when speeding up the process of development has become imperative. The plan adopts the drive towards a knowledge based economy 
through focusing on human development and education. The main challenges of education are improving enrolment rates, reducing dropout rates at all levels of education, and enabling education to meet the demand of labor market. As a step towards the demand of labor market, new programs are initiated with focusing on important fields and areas besides development of curriculum. In addition, to absorbing the graduates from the secondary schools, new universities have been set up distributed in the different regions of the Kingdom. Great efforts have been undertaken during the 9th development plan to develop those new universities and to equip them to carry out the basic educational duties assigned to them, together with other endeavors aimed to further develop universities already established, and to concentrate on programs of quality, excellence, creativity, and partnership with distinguished international educational institutions. The ministry seeks to realize those strategic goals in accordance with the following points [20]:

(i) Admission capacity for institutions of higher education in the Kingdom.

(ii) Matching university education with the requirements of national development.

(iii) Quality in higher education.

(iv) Development of scholarly research serving the goals of development.

(v) Building international strategic partnerships in the realm of higher education.

(vi) Firmly establishing and strengthening modern administration in higher education sectors.

(vii) Diversification of resources in financing higher education.

In the future plan for higher education in Saudi Arabia, higher education is designed and evaluated in relation to the overall national development plan and is considered essential for evaluating a skilled workforce for its socioeconomic development, promoting research and development, and maintaining its distinctive cultural heritage. Since 2006, and subsequently as a part of the Future Plan for Higher Education in Saudi Arabia "AAFAQ" [21], a variety of policies were designed and are being implemented at promoting excellence in the higher education. The main designed policies are

(i) expanding university capacities,

(ii) study-abroad policy,

(iii) National Commission for Assessment and Accreditation.

The main objectives stated in specified numerical values in the 9th national development plan 2010-2014 and that in the future plan for higher education in Saudi Arabia can be stated in the following goals.

Goal Number 1. The KSA Development Plan aims to enroll in its first year, 2010, about 315,300 students and increase this number gradually to about 375,300 students in its last year, 2014 , with an average annual growth rate $p_{r}^{y}=4.5 \%$.

Goal Number 2. The percentage of the total number of students enrolled in science and technology programs to the total number of students enrolled in higher education in the same year is $p_{s}^{y}=60 \%$.

Goal Number 3-1. The percentage of total enrollment in higher education regardless of age to the total population in the age group of $18-23$ years in the same year is $p_{p}^{y}=50 \%$.

Goal Number 3-2. The accepted percentage in higher education from high school graduates in the same year is $p_{h}^{y}=55 \%$.

Goal Number 4. The percentage of the total number of students in each discipline of university education to the total faculty $(F)$ in that specialty is as follows:

Medicine $(M) \beta_{M}^{y}=10: 1$.

Science and engineering $(S) \beta_{S}^{y}=17: 1$.

Arts $(A) \beta_{A}^{y}=22: 1$.

Total university $(U) \beta_{U}^{y}=20: 1$.

Specialties will be denoted by " $m$ " for a college in a medicine programs, " $s$ " for a college in the science and engineering programs, and " $a$ " for a college in the arts program. " $u$ " will denote a college in the set of all colleges in a university $U$ :

$M=$ the set of colleges in the medicine program in a university,

$S=$ the set of colleges in the science and engineering program in a university,

$A=$ the set of colleges in the arts program in a university,

$U=$ the set of all colleges in all programs in a university.

Goal Number 5 (Budget Constraint). Let $B_{U}^{y}$ be the total budget of the university " $U$ " that should not exceed a certain limit at any year " $y$ " within a planning horizon.

Goal Number 6-1. The plan aims at increasing the number of graduates from 240,800 to about 318,300 , with an average annual growth rate $q_{r}^{y}=7.2 \%$.

Goal Number 6-2. Percentage of students who have completed their studies in a given year to the total number of students enrolled in universities five years before that year is $q_{d}^{y}=85 \%$.

\section{GP Model for the Admission Problem}

In general mathematical programming methods, the objective function is measured in one dimension only. It is not possible for linear programming, for example, to have multiple goals unless they are all measured in the same units, and this is a highly unusual situation. 
Organizations often have more than one goal; they may want to achieve several contradictory goals at the same time. It is not always possible to satisfy every goal in full extent, so attempts are made to reach a satisfactory level of the stated multiple objectives.

The main difference is in the objective function where GP tries to minimize the deviations between goals and what we can actually achieve within the given constraints.

The mathematical model will cover the main objectives stated in the current KSA Development Plan and that stated in KSA Higher Education Strategic Plan (AAFAQ) for the next 25 years. It will be restricted also to the budget and staff constraints as problem resources. The data are extracted from the formal organization references and their published statistical books [22-25].

4.1. Decision Variables. Let: $x_{i, j, k}^{y}=x_{\text {status, gender, program }}^{\text {yea of the plan }}=$ No of students, where: $y=1,2,3,4$, and 5 will denote the year of the upcoming 5 -year plan, where $y=1$ is the first year in that plan and $y=2$ is the second year and so on, and $y=0,-1,-2$, -3 , and -4 will denote the years of the previous 5 -year plan, where $y=0$ indicates the current (last) year in the previous plan, which is one year before the first year of the upcoming plan. $y=-1$ is the 4 th year of the last plan and $y=-2$ is the 3rd year of the last plan and so on. $i$ is status that will be denoted by $E$ for enrolled and $G$ for graduated, $j$ is gender that will be denoted by $b$ for boys and $g$ for girls, $k$ is a college belonging to a program $K$, where " $m$ " denotes medicine, " $s$ " science and engineering, and " $a$ " arts.

4.2. Problem Goals. The Kingdom Development Plan and AAFAQ Project objectives are formulated to represent the mathematical model goals as follows.

Let

$$
\begin{aligned}
& d_{n}^{y-}=\text { underachievement of the } n \text {th target in year } y, \\
& d_{n}^{y+}=\text { overachievement of the } n \text {th target in year } y,
\end{aligned}
$$

where $n$ is the number of the targets to be fulfilled.

First Goal. Consider

$$
\begin{array}{r}
\frac{\left(\sum_{u \in U} \mathbf{x}_{E b u}^{y}-\sum_{u \in U} \mathbf{x}_{E b u}^{y-1}\right)}{\sum_{u \in U} \mathbf{x}_{E b u}^{y-1}}+d_{1}^{-}-d_{1}^{+}=p_{r}^{y}, \\
\frac{\left(\sum_{u \in U} \mathbf{x}_{E g u}^{y}-\sum_{u \in U} \mathbf{x}_{E g u}^{y-1}\right)}{\sum_{u \in U} \mathbf{x}_{E g u}^{y-1}}+d_{2}^{-}-d_{2}^{+}=p_{r}^{y}, \\
y=1,2,3,4,5 .
\end{array}
$$

Second Goal. Consider

$$
\begin{array}{r}
\frac{\left(\sum_{m \in M} \mathbf{x}_{E b m}^{y}+\sum_{e \in E} \mathbf{x}_{E b s}^{y}\right)}{\sum_{u \in U} \mathbf{x}_{E b u}^{y}}+d_{3}^{y-}-d_{3}^{y+}=p_{s}^{y}, \\
\frac{\left(\sum_{m \in M} \mathbf{x}_{E g m}^{y}+\sum_{e \in E} \mathbf{x}_{E g s}^{y}\right)}{\sum_{u \in U} \mathbf{x}_{E g u}^{y}}+d_{4}^{y-}-d_{4}^{y+}=p_{s}^{y}, \\
y=1,2,3,4,5 .
\end{array}
$$

Third Goal. Consider

$$
\begin{array}{r}
\sum_{j=b, g} \sum_{k=U} \mathbf{x}_{E, j, k}^{y}+d_{5}^{y-}-d_{5}^{y+} \\
=\max \left[\left(\frac{1}{5} \cdot p_{p}^{y} \cdot \propto^{y} \cdot N_{C}^{y-1}\right),\left(p_{h}^{y} \cdot \propto^{y} \cdot N_{H}^{y-1}\right)\right], \\
y=1,2,3,4,5,
\end{array}
$$

where $p_{p}^{y}=$ the percentage of total enrollment in higher education regardless of age, to the total population in the age group of 18-23 years in year $y . \propto^{y}=$ the portion admitted to a specific university $U$ to all the universities in the Kingdom in a year $y \cdot N_{C}^{y}=$ population of Saudi Arabia in the age of 18-23 years in year $y \cdot p_{h}^{y}=$ the accepted percentage in higher education from high school graduates in year $y . N_{H}^{y}=$ high school graduates in a year $y$ that will be decreased by the number of boys for bachelor scholarships abroad $\left(N_{b}^{y-1}\right)$ and the number of girls for bachelor scholarships abroad $\left(N_{g}^{y-1}\right)$.

Fourth Goal. Consider

$$
\begin{aligned}
& \sum_{m \in M} \mathbf{x}_{E b m}^{y}+d_{6}^{1-}-d_{6}^{1+}=\frac{\mathbf{1}}{\mathbf{t}_{\mathbf{M}}} \cdot \beta_{M}^{y} \cdot F_{b M}^{y}, \\
& \sum_{m \in M} \mathbf{x}_{E g m}^{y}+d_{7}^{1-}-d_{7}^{1+}=\frac{\mathbf{1}}{\mathbf{t}_{\mathbf{M}}} \cdot \beta_{M}^{y} \cdot F_{g M}^{y}, \\
& \sum_{s \in S} \mathbf{x}_{E b s}^{y}+d_{8}^{1-}-d_{8}^{1+}=\frac{\mathbf{1}}{\mathbf{t}_{\mathbf{s}}} \cdot \beta_{S}^{y} \cdot F_{b S}^{y}, \\
& \sum_{s \in S} \mathbf{x}_{E g s}^{y}+d_{9}^{1-}-d_{9}^{1+}=\frac{\mathbf{1}}{\mathbf{t}_{\mathbf{s}}} \beta_{S}^{y} \cdot F_{g S}^{y}, \\
& \sum_{a \in A} \mathbf{x}_{E b a}^{y}+d_{10}^{1-}-d_{10}^{1+}=\frac{\mathbf{1}}{\mathbf{t}_{\mathbf{A}}} \cdot \beta_{A}^{y} \cdot F_{b A}^{y}, \\
& \sum_{a \in A} \mathbf{x}_{E g a}^{y}+d_{11}^{1-}-d_{11}^{1+}=\frac{\mathbf{1}}{\mathbf{t}_{\mathbf{A}}} \cdot \beta_{A}^{y} \cdot F_{g A}^{y}, \\
& \sum_{u \in U} \mathbf{x}_{E b u}^{y}+d_{12}^{1-}-d_{12}^{1+}=\frac{\mathbf{1}}{\mathbf{t}_{\mathbf{U}}} \cdot \beta_{U}^{y} \cdot F_{b U}^{y}, \\
& \sum_{u \in U} \mathbf{x}_{E g u}^{y}+d_{13}^{1-}-d_{13}^{1+}=\frac{\mathbf{1}}{\mathbf{t}_{\mathbf{U}}} \cdot \beta_{U}^{y} \cdot F_{g U}^{y}, \\
& y=1,2,3,4,5,
\end{aligned}
$$


where $F_{j, K}^{y}=$ number of faculty members for gender $j$ in specialty $K, j=b, g$ and $K=M, S, A$, and $U$, and $t_{k}=$ number of years in a program $K$.

Fifth (Budget) Goal. Since the maximum available budget in a certain year $y$ is limited, then the total number of students enrolled should not exceed this allocated budget.

Let

$c_{U}^{y}=$ cost per student in the university in a year $y$,

$B_{U}^{y}=$ maximum budget for the university in a year $y$, $y=1,2,3,4,5$.

Then

$$
\left(x_{E b U}^{y}+x_{E g U}^{y}\right) \cdot c_{U}^{y}+d_{14}^{1-}-d_{14}^{1+}=B_{U}^{y},
$$

$$
y=1,2,3,4,5 \text {. }
$$

\section{Sixth Goal (Graduation Constraint). Consider}

$$
\begin{gathered}
\sum_{m \in M} \mathbf{x}_{G b m}^{y} \geq \max \left[q_{d}^{y} \cdot \sum_{m \in M} \mathbf{x}_{E b m}^{y-5},\left(1+q_{r}^{y}\right) \cdot \sum_{m \in M} \mathbf{x}_{G b m}^{y-1}\right], \\
\sum_{s \in S} \mathbf{x}_{G b s}^{y} \geq \max \left[q_{d}^{y} \cdot \sum_{s \in S} \mathbf{x}_{E b s}^{y-4},\left(1+q_{r}^{y}\right) \cdot \sum_{s \in S} \mathbf{x}_{G b s}^{y-1}\right], \\
\sum_{a \in A} \mathbf{x}_{G b a}^{y} \geq \max \left[q_{d}^{y} \cdot \sum_{a \in A} \mathbf{x}_{E b a}^{y-3},\left(1+q_{r}^{y}\right) \cdot \sum_{a \in A} \mathbf{x}_{G b a}^{y-1}\right], \\
\sum_{m \in M} \mathbf{x}_{G g m}^{y} \geq \max \left[q_{d}^{y} \cdot \sum_{m \in M} \mathbf{x}_{E g m}^{y-5},\left(1+q_{r}^{y}\right) \cdot \sum_{m \in M} \mathbf{x}_{G g m}^{y-1}\right], \\
\sum_{s \in S} \mathbf{x}_{G g s}^{y} \geq \max \left[q_{d}^{y} \cdot \sum_{s \in S} \mathbf{x}_{E b s}^{y-4},\left(1+q_{r}^{y}\right) \cdot \sum_{s \in S} \mathbf{x}_{G g s}^{y-1}\right], \\
\sum_{a \in A} \mathbf{x}_{G g a}^{y} \geq \max \left[q_{d}^{y} \cdot \sum_{a \in A} \mathbf{x}_{E b a}^{y-3},\left(1+q_{r}^{y}\right) \cdot \sum_{m \in M} \mathbf{x}_{G g a}^{y-1}\right], \\
y=1,2,3,4,5 .
\end{gathered}
$$

4.3. Logic Constraints. The total number of students in the university is equal to the total number of students in all the specialties, medicine, science and engineering, and arts, and this is applied for both boys and girls as follows:

$$
\begin{aligned}
\sum_{u \in U} \mathbf{x}_{E b u}^{y} & =\sum_{m \in M} \mathbf{x}_{E b m}^{y}+\sum_{e \in E} \mathbf{x}_{E b s}^{y}+\sum_{a \in A} \mathbf{x}_{E b a}^{y} \\
\sum_{u \in U} \mathbf{x}_{E g u}^{y} & =\sum_{m \in M} \mathbf{x}_{E g m}^{y}+\sum_{e \in E} \mathbf{x}_{E g s}^{y}+\sum_{a \in A} \mathbf{x}_{E g a}^{y} \\
\sum_{u \in U} \mathbf{x}_{E b u}^{y} \geq \sum_{u \in U} \mathbf{x}_{E b u}^{y-1}, & \\
\sum_{u \in U} \mathbf{x}_{E g u}^{y} \geq \sum_{u \in U} \mathbf{x}_{E g u}^{y-1}, & y=1,2,3,4,5 .
\end{aligned}
$$

4.4. Objective Function. Once all goals and constraints are identified, higher management in the institution should analyze each goal to see if underachievement or overachievement of that goal is an acceptable situation:

(i) If overachievement or underachievement is acceptable, the appropriate corresponding deviation variable is eliminated from the objective function.

(ii) If management seeks to attain a goal exactly, both deviation variables must appear in the objective function.

Typically goals set by management can be achieved only at the expense of other goals. A hierarchy of importance needs to be established so that higher-priority goals are satisfied before lower-priority goals are addressed. Priorities $\left(P_{i}\right.$ 's) are assigned to each deviational variable with the ranking so that $P 1$ is the most important goal, $P 2$ the next most important, P3 the third, and so on.

In the problem under investigation, it is considered that the goals and system constraints related to the planning year number 1 have a higher priority than all the other years, and those related to the second year are higher than the third, fourth, and fifth, and so on. The same weights will be given to all the goals in the same priority level. Accordingly the problem will be divided into several problems; each one is related only to one planning year. The results obtained from each priority will be considered as constraints for the other priority levels.

So, the objective function is formulated as follows:

$$
\text { Minimize } \begin{aligned}
f(x)= & d_{1}^{y-}+d_{2}^{y-}+d_{3}^{y-}+d_{3}^{y+}+d_{4}^{y-}+d_{4}^{y+} \\
& +d_{5}^{y-}+d_{6}^{y+}+d_{7}^{y+}+d_{8}^{y+}+d_{9}^{y+} \\
& +d_{10}^{y+}+d_{11}^{y+}+d_{12}^{y+}+d_{13}^{y+}+d_{14}^{y+} .
\end{aligned}
$$

\section{King Abdulaziz University as a Case Study}

Since each year has its specific data, the model will be formulated and solved initially for the first year. The obtained results will be given as input data for the second year and so on till reaching the last year of the 5 th year plan.

5.1. Mathematical Model for the First Year. Substituting $y=$ 1 , the mathematical model for the first priority level can be formulated as follows:

$$
\begin{aligned}
\text { Minimize } f(x)= & d_{1}^{1-}+d_{2}^{1-}+d_{3}^{1-}+d_{3}^{1+}+d_{4}^{1-}+d_{4}^{1+} \\
& +d_{5}^{1-}+d_{6}^{1+}+d_{7}^{1+}+d_{8}^{1+}+d_{9}^{1+} \\
& +d_{10}^{1+}+d_{11}^{1+}+d_{12}^{1+}+d_{13}^{1+}+d_{14}^{1+}
\end{aligned}
$$

Constraints. In order to minimize the number of deviation variables, each overachievement or underachievement deviation variable that is not included in the objective function and can take a positive value different from 0 will be omitted from 
its corresponding constraint and the sign of the constraint will be adjusted correspondingly as follows.

Goal 1. Consider

$$
\begin{aligned}
& \frac{\left(\mathbf{x}_{E b U}^{1}-\mathbf{x}_{E b U}^{0}\right)}{\mathbf{x}_{E b U}^{0}}+d_{1}^{1-} \geq p_{r}^{1}, \\
& \frac{\left(\mathbf{x}_{E g U}^{1}-\mathbf{x}_{E g U}^{0}\right)}{\mathbf{x}_{E g U}^{0}}+d_{2}^{1-} \geq p_{r}^{1} .
\end{aligned}
$$

Logic Constraints. Consider

$$
\begin{aligned}
& \mathbf{x}_{E b U}^{1} \geq \mathbf{x}_{E b U}^{0}, \\
& \mathbf{x}_{E g U}^{1} \geq \mathbf{x}_{E g U}^{0} .
\end{aligned}
$$

Goal 2. Consider

$$
\begin{aligned}
& \frac{\left(\mathbf{x}_{E b M}^{1}+\mathbf{x}_{E b S}^{1}\right)}{\mathbf{x}_{E b U}^{1}}+d_{3}^{1-}-d_{3}^{1+}=p_{s}^{1}, \\
& \frac{\left(\mathbf{x}_{E g M}^{1}+\mathbf{x}_{E g S}^{1}\right)}{\mathbf{x}_{E g U}^{1}}+d_{4}^{1-}-d_{4}^{1+}=p_{s}^{1} .
\end{aligned}
$$

Goal 3. Consider

$$
\begin{aligned}
& \left(\mathbf{x}_{E b U}^{1}+\mathbf{x}_{E g U}^{1}\right)+d_{5}^{1-} \\
& \quad \geq \max \left[\left(\frac{1}{5} \cdot p_{p}^{1} \cdot \alpha^{1} \cdot N_{C}^{0}\right),\left(p_{h}^{y} \cdot \propto^{1} \cdot N_{H}^{0}\right)\right] .
\end{aligned}
$$

Goal 4. Consider

$$
\begin{aligned}
& \mathbf{x}_{E b M}^{1}-d_{6}^{1+} \leq \frac{\mathbf{1}}{\mathbf{5}} \cdot \beta_{M}^{1} \cdot F_{b M}^{1}, \\
& \mathbf{x}_{E g M}^{1}-d_{7}^{1+} \leq \frac{\mathbf{1}}{\mathbf{5}} \cdot \beta_{M}^{1} \cdot F_{g M}^{1}, \\
& \mathbf{x}_{E b S}^{1}-d_{8}^{1+} \leq \frac{\mathbf{1}}{\mathbf{4}} \cdot \beta_{E}^{1} \cdot F_{b S}^{1} \\
& \mathbf{x}_{E g S}^{1}-d_{9}^{1+} \leq \frac{\mathbf{1}}{\mathbf{4}} \cdot \beta_{E}^{1} \cdot F_{g S}^{1} \\
& \mathbf{x}_{E b A}^{1}-d_{10}^{1+} \leq \frac{\mathbf{1}}{\mathbf{3}} \cdot \beta_{A}^{1} \cdot F_{b A}^{1}, \\
& \mathbf{x}_{E b A}^{1}-d_{11}^{1+} \leq \frac{\mathbf{1}}{\mathbf{3}} \beta_{A}^{1} \cdot F_{b A}^{1}, \\
& \mathbf{x}_{E b U}^{1}-d_{12}^{1+} \leq \frac{\mathbf{1}}{\mathbf{5}} \beta_{U}^{1} \cdot F_{b U}^{1} \\
& \mathbf{x}_{E g U}^{1}-d_{13}^{1+} \leq \frac{\mathbf{1}}{\mathbf{5}} \beta_{U}^{1} \cdot F_{g U}^{1} .
\end{aligned}
$$

Logic Constraints. Consider

$$
\begin{aligned}
& \mathbf{x}_{E b U}^{1}=\mathbf{x}_{E b M}^{1}+\mathbf{x}_{E b S}^{1}+\mathbf{x}_{E b A}^{1}, \\
& \mathbf{x}_{E g U}^{1}=\mathbf{x}_{E g M}^{1}+\mathbf{x}_{E g S}^{1}+\mathbf{x}_{E g A}^{1} .
\end{aligned}
$$

Goal 5. Consider

$$
\left(\mathbf{x}_{E b U}^{1}+\mathbf{x}_{E g U}^{1}\right) \cdot c_{U}^{1}-d_{14}^{1+} \leq B_{U}^{1} .
$$

Goal 6. Consider

$$
\begin{aligned}
& \mathbf{x}_{G b M}^{1} \geq \max \left[q_{d}^{1} \cdot \mathbf{x}_{E b M}^{-5},\left(1+q_{r}^{1}\right) \cdot \mathbf{x}_{G b M}^{0}\right], \\
& \mathbf{x}_{G b S}^{1} \geq \max \left[q_{d}^{1} \cdot \mathbf{x}_{E b S}^{-5},\left(1+q_{r}^{1}\right) \cdot \mathbf{x}_{G b S}^{0}\right], \\
& \mathbf{x}_{G b A}^{1} \geq \max \left[q_{d}^{1} \cdot \mathbf{x}_{E b A}^{-5},\left(1+q_{r}^{1}\right) \cdot \mathbf{x}_{G b A}^{0}\right], \\
& \mathbf{x}_{G g M}^{1} \geq \max \left[q_{d}^{1} \cdot \mathbf{x}_{E b M}^{-5},\left(1+q_{r}^{1}\right) \cdot \mathbf{x}_{G g M}^{0}\right], \\
& \mathbf{x}_{G g S}^{1} \geq \max \left[q_{d}^{1} \cdot \mathbf{x}_{E b S}^{-5},\left(1+q_{r}^{1}\right) \cdot \mathbf{x}_{G g S}^{0}\right], \\
& \mathbf{x}_{G g A}^{1} \geq \max \left[q_{d}^{1} \cdot \mathbf{x}_{E b A}^{-5},\left(1+q_{r}^{1}\right) \cdot \mathbf{x}_{G g A}^{0}\right], \\
& \mathbf{x}_{G b U}^{1}=\mathbf{x}_{G b M}^{1}+\mathbf{x}_{G b S}^{1}+\mathbf{x}_{G b A}^{1}, \\
& \mathbf{x}_{G g U}^{1}=\mathbf{x}_{G g M}^{1}+\mathbf{x}_{G g S}^{1}+\mathbf{x}_{G g A}^{1} .
\end{aligned}
$$

The data collected from King Abdulaziz University is portrayed in Table 1 .

\subsection{Discussions and Different Scenarios}

(i) It can be noticed that the mathematical model contains two distinct parts; one is related to the enrollment process while the other is related to the graduation process. The enrollment part is stated in the first 5 goals, while the graduation part is specified only in the 6th constraint.

(ii) The decision variables related to the graduation process are not included in the objective function or in the constraints. Since these decision variables are not restricted, then related constraints numbers (25)-(30) are completely satisfied without any effect on other parts of the enrollment process.

(iii) The problem of student enrollment is solved with data representing the first year of the national development plan for the Kingdom of Saudi Arabia (2015) applied for King Abdulaziz University with different scenarios representing the priority levels for different goals to open the scope for the decision maker to choose according to his preference. The results are shown in Table 2.

Scenario Number 1 . In the first scenario, all the 5 enrollment goals are given equal weights of 1 in the objective function. Some of the goals are satisfied in the optimum solution for this scenario, while some others are over- or underachieved:

(i) Constraint number (11) for enrollment is the active and binding conditions.

(ii) Constraints numbers (12), (17), (18), and (20) are exactly satisfied, so the first part of goal number 2 and some parts of goal number 4 are satisfied. 
TABLE 1: Relevant data related to King Abdulaziz University.

\begin{tabular}{lc}
\hline Abbreviation & Value \\
\hline$x_{E b U}^{0}$ & 21,927 \\
$x_{E g U}^{0}$ & 17,607 \\
$\propto^{1}$ & 0.147 \\
$N_{C}^{0}$ & $1,297,000$ \\
$N_{H}^{0}$ & 404,742 \\
$N_{b}^{0}$ & 20,644 \\
$N_{g}^{0}$ & 7,777 \\
$F_{b M}^{1}$ & 1,834 \\
$F_{g M}^{1}$ & 1,188 \\
$F_{b S}^{1}$ & 8,734 \\
$F_{g S}^{1}$ & 6,392 \\
$F_{b A}^{1}$ & 6,204 \\
$F_{g A}^{1}$ & 8,265 \\
$F_{b U}^{1}$ & 15,272 \\
$F_{g U}^{1}$ & 12,900 \\
$B_{U}^{1}$ & $4,778.5$ millions \\
$c_{U}^{1}$ & 56,259 \\
$x_{E b M}^{-5}$ & 236 \\
$x_{G b M}^{0}$ & 367 \\
$x_{E b S}^{-4}$ & 1,969 \\
$x_{G b S}^{0}$ & 5,188 \\
$x_{E b A}^{-3}$ & 9,150 \\
$x_{G b A}^{0}$ & 2,730 \\
$x_{E g M}^{-5}$ & 318 \\
$x_{G g M}^{0}$ & 626 \\
$x_{E g S}^{-4}$ & 5,377 \\
$x_{G g S}^{0}$ & 4,418 \\
$x_{E g A}^{-3}$ & 5,981 \\
$x_{G g A}^{0}$ & 3,291 \\
\hline &
\end{tabular}

(iii) Constraints numbers (10), (13), and (24) are underachieved.

(iv) Constraints numbers (14), (15), (16), (19), (21), and (22) are overachieved.

(v) So, excess budget and smaller number of enrollment students than the required targets in some goals resulted.

Scenario Number 2. In the second scenario, goal number 1 is given the highest priority while constraint number (11) becomes redundant. This is done by considering the related underachievement deviation variables in (10) equal to zero both in the objective function and in the constraints. In this scenario, the following results are obtained:

(i) Constraints numbers (10), (12), (17), (18), and (20) are exactly satisfied.

(ii) Constraints numbers (13) and (24) are underachieved.

(iii) Constraints numbers (14), (15), (16), (19), (21), and (22) are overachieved.
Scenario Number 3. In this scenario, goal number 2 is given the highest priority while keeping satisfying the first goal. This is done by considering the related overachievement and underachievement deviation variables equal to zero both in the objective function and in the constraints. In this scenario, the following results are obtained:

(i) Constraints numbers (10), (12), (13), (17), and (18) are exactly satisfied.

(ii) Constraints numbers (20) and (24) are underachieved.

(iii) Constraints numbers (14), (15), (16), (19), (21), and (22) are overachieved.

In the above three scenarios, it is noticed that goal number 3 is satisfied while corresponding constraint number (14) is overachieved.

Scenario Number 4 . In this scenario, goal number 4 is given the highest priority over other conflicting goals 1 and 3 . This is done by considering the related overachievement deviation variables equal to zero both in the objective function and in the constraints while contradicting constraint (11) is relaxed. In this scenario, the following results are obtained:

(i) Constraints numbers (12), (13), (16), (17), (21), and (22) are exactly satisfied.

(ii) Constraints numbers (10), (14), (15), (18), (19), (20), and (24) are underachieved.

(iii) None of the constraints is overachieved.

(iv) It is noticed that only part of the constraints of goal number 4 is satisfied since some of these constraints are conflicting with the remaining constraints.

\section{Proposed Differential Evolution Approach and Problem Solution}

It can be seen that the proposed GPM contains nonlinear constraints, involves numerous amounts of integer variables, and is not as simple as the linear GP model with continuous variables. Therefore, a novel constrained optimization based on modified differential evolution algorithm named COMDE (Mohamed and Sabry, 2012) [26] is used to solve the proposed nonlinear integer GP problem. Differential evolution, DE, has been recently receiving great attention and is successfully applied in many research fields in the last decade (Das and Suganthan, 2011) [27]. However, to the best of our knowledge, this is the first time to use DE in solving admission problems in higher education.

6.1. Differential Evolution (DE). Differential evolution (DE) is a stochastic population-based search method, proposed by Storn and Price [28]. DE is relatively recent EAs for solving real-parameter optimization problems [28]. DE has many advantages including simplicity of implementation, reliability, and robustness, and in general it is considered as an effective global optimization algorithm [29]. In this paper, the scheme which can be classified using the notation 
TABLE 2: Optimal values of nonzero decision variables for different scenarios.

\begin{tabular}{|c|c|c|c|c|c|c|}
\hline Number & Decision variable & Abbreviation & Scenario 1 & Scenario 2 & Scenario 3 & Scenario 4 \\
\hline 1 & $x_{E b U}^{1}$ & $x_{1}$ & 21,927 & 22,914 & 22,914 & 15,272 \\
\hline 2 & $x_{E g U}^{1}$ & $x_{2}$ & 17,607 & 18,400 & 18,400 & 12,900 \\
\hline 3 & $x_{E b M}^{1}$ & $x_{3}$ & 4,423 & 5014 & 5014 & 429 \\
\hline 4 & $x_{E b E}^{1}$ & $x_{4}$ & 8,734 & 8,734 & 8,734 & 8,734 \\
\hline 5 & $x_{E g M}^{1}$ & $x_{5}$ & 2,950 & 3743 & 4,648 & 1,188 \\
\hline 6 & $x_{E g E}^{1}$ & $x_{6}$ & 6,392 & 6,392 & 6,392 & 6,178 \\
\hline 7 & $x_{E b A}^{1}$ & $x_{7}$ & 8,770 & 9,166 & 9,166 & 6,108 \\
\hline 8 & $x_{E g A}^{1}$ & $x_{8}$ & 8,265 & 8,265 & 7,360 & 5,534 \\
\hline 9 & $d_{1}^{1-}$ & $x_{9}$ & 987 & 0 & 0 & 7,642 \\
\hline 10 & $d_{2}^{1-}$ & $x_{10}$ & 793 & 0 & 0 & 5,500 \\
\hline 11 & $d_{4}^{1-}$ & $x_{13}$ & 1222 & 905 & 0 & 0 \\
\hline 12 & $d_{5}^{1-}$ & $x_{15}$ & 0 & 0 & 0 & 374 \\
\hline 13 & $d_{6}^{1+}$ & $x_{16}$ & 2,589 & 3,180 & 3,180 & \\
\hline 14 & $d_{7}^{1+}$ & $x_{17}$ & 1,762 & 2,555 & 3,460 & \\
\hline 15 & $d_{10}^{1+}$ & $x_{20}$ & 2,566 & 2,962 & 2,962 & \\
\hline 16 & $d_{12}^{1+}$ & $x_{22}$ & 6,655 & 7,642 & & \\
\hline 17 & $d_{13}^{1+}$ & $x_{23}$ & 4,707 & 5,500 & 5,500 & \\
\hline 18 & $x_{G b M}^{1}$ & $x_{25}$ & \multicolumn{4}{|c|}{394} \\
\hline 19 & $x_{G b S}^{1}$ & $x_{26}$ & \multicolumn{4}{|c|}{5,562} \\
\hline 20 & $x_{G b A}^{1}$ & $x_{27}$ & \multicolumn{4}{|c|}{2,927} \\
\hline 21 & $x_{G g M}^{1}$ & $x_{28}$ & \multicolumn{4}{|c|}{672} \\
\hline 22 & $x_{G g S}^{1}$ & $x_{29}$ & \multicolumn{4}{|c|}{4,737} \\
\hline 23 & $x_{G g A}^{1}$ & $x_{30}$ & \multicolumn{4}{|c|}{5084} \\
\hline 24 & $x_{G b U}^{1}$ & $x_{31}$ & \multicolumn{4}{|c|}{8,883} \\
\hline \multirow[t]{2}{*}{25} & $x_{G g U}^{1}$ & $x_{32}$ & \multicolumn{4}{|c|}{10,493} \\
\hline & $z$ & & 21,281 & 22,744 & 22,744 & 13,516 \\
\hline
\end{tabular}

as $\mathrm{DE} / \mathrm{rand} / 1 /$ bin strategy is used $[30,31]$. This strategy is most often used in practice. A set of $D$ optimization parameters is called an individual, which is represented by a $D$-dimensional parameter vector. A population consists of NP parameter vectors $x_{i}^{G}, i=1,2, \ldots$, NP. $G$ denotes one generation. NP is the number of members in a population. It does not change during the evolution process. The initial population is chosen randomly with uniform distribution in the search space. DE has three operators: mutation, crossover, and selection. The crucial idea behind DE is a scheme for generating trial vectors. Mutation and crossover operators are used to generate trial vectors, and the selection operator then determines which of the vectors will survive into the next generation [31-33].

6.1.1. Initialization. In order to establish a starting point for the optimization process, an initial population must be created. Typically, each decision parameter in every vector of the initial population is assigned a randomly chosen value from the boundary constraints:

$$
x_{i j}^{0}=l_{j}+\operatorname{rand}_{j} *\left(u_{j}-l_{j}\right),
$$

where rand $_{j}$ denotes a uniformly distributed number in the range of $[0,1]$, generating a new value for each decision parameter. $l_{i}$ and $u_{i}$ are the lower and upper bounds for the $j$ th decision parameter, respectively [28].

6.1.2. Mutation. For each target vector $x_{i}^{G}$, a mutant vector $v$ is generated according to the following:

$$
v_{i}^{G+1}=x_{r_{1}}^{G}+F *\left(x_{r_{2}}^{G}-x_{r_{3}}^{G}\right), \quad r_{1} \neq r_{2} \neq r_{3} \neq i
$$

with randomly chosen indices and $r_{1}, r_{2}, r_{3} \in\{1,2, \ldots, \mathrm{NP}\}$.

Note that these indices have to be different from each other and from the running index $i$ so that NP must be at least four. $F$ is a real number to control the amplification of the difference vector $\left(x_{r_{2}}^{G}-x_{r_{3}}^{G}\right)$. According to [29], the range of $F$ is in $[0,2]$. If a component of a mutant vector goes off the search space, that is, if a component of a mutant vector violates the boundary constraints, then the new value of this component is generated using (33).

6.1.3. Crossover. The target vector is mixed with the mutated vector, using the following scheme, to yield the trial vector $u$ :

$$
u_{i j}^{G+1}= \begin{cases}v_{i j}^{G+1}, & \operatorname{rand}(j) \leq \mathrm{CR} \text { or } j=\operatorname{rand} n(i), \\ x_{i j}^{G}, & \operatorname{rand}(j)>\mathrm{CR}, j \neq \operatorname{rand} n(i),\end{cases}
$$




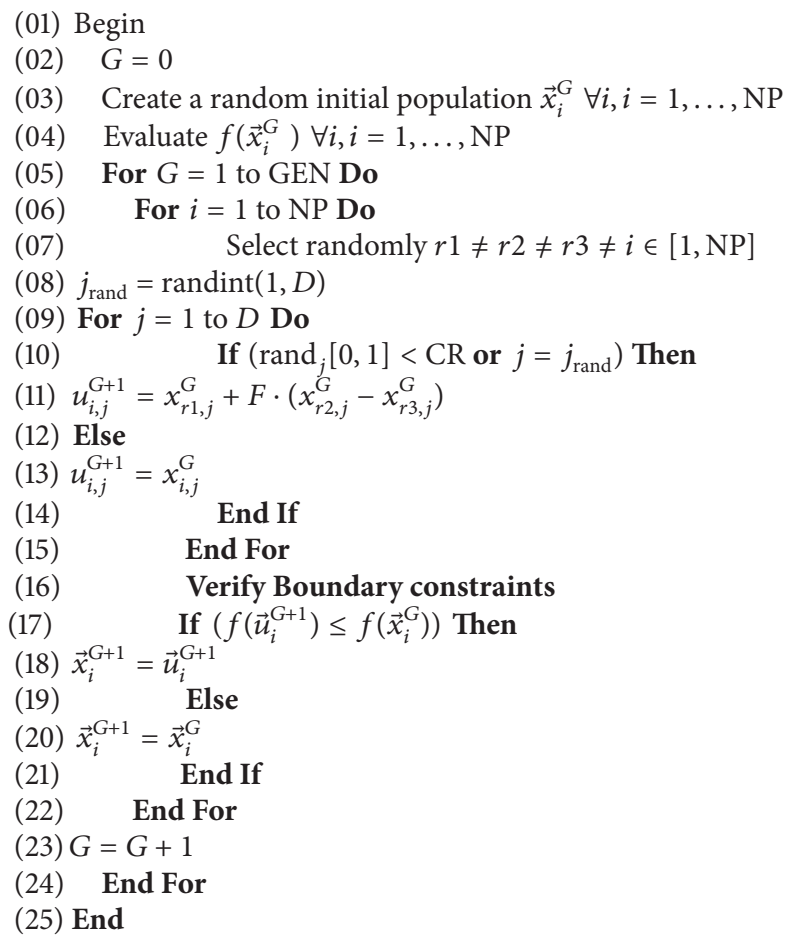

Algorithm 1: Description of standard DE algorithm. rand[0,1) is a function that returns a real number between 0 and 1 ; randint (min, max) is a function that returns an integer number between min and max. NP, GEN, CR, and $F$ are user-defined parameters. $D$ is the dimensionality of the problem.

where $j=1,2, \ldots, D$ and $\operatorname{rand}(j) \in[0,1]$ is the $j$ th evaluation of a uniform random generator number. $\mathrm{CR} \in[0,1]$ is the crossover probability constant, which has to be determined by the user. $\operatorname{rand} n(i) \in\{1,2, \ldots, D\}$ is a randomly chosen index which ensures that $u_{i}^{G+1}$ gets at least one element from $v_{i}^{G+1}$.

6.1.4. Selection. DE adapts a greedy selection strategy. If and only if the trial vector $u_{i}^{G+1}$ yields a better fitness function value than $x_{i}^{G}$, then $u_{i}^{G+1}$ is set to $x_{i}^{G+1}$. Otherwise, the old value $x_{i}^{G}$ is retained. The selection scheme is as follows (for a minimization problem):

$$
x_{i}^{G+1}= \begin{cases}u_{i}^{G+1}, & f\left(u_{i}^{G+1}\right)<f\left(x_{i}^{G}\right), \\ x_{i}^{G}, & f\left(u_{i}^{G+1}\right) \geq f\left(x_{i}^{G}\right) .\end{cases}
$$

A detailed description of standard DE algorithm is given in Algorithm 1.

6.2. Constrained Optimization Based on Modified Differential Evolution Algorithm (COMDE). All evolutionary algorithms, including DE, are stochastic population-based search methods. Accordingly, there is no guarantee that the global optimal solution will be reached consistently. Furthermore, they are not originally designed to solve constrained optimization problems. Nonetheless, adjusting control parameters such as the scaling factor, the crossover rate, and the population size alongside developing an appropriate mutation scheme and coupling with suitable and effective constraint handling techniques can considerably improve the search capability of DE algorithms [26]. Therefore, in the proposed algorithm, a new directed mutation rule, based on the weighted difference vector between the best and the worst individuals at a particular generation, is introduced. The new directed mutation rule is combined with the modified basic mutation strategy DE/rand/1/bin, where only one of the two mutation rules is applied with the probability of 0.5 . The proposed mutation rule is shown to enhance the local search ability of the basic differential evolution (DE) and to get a better trade-off between convergence rate and robustness. Two new scaling factors are introduced as uniform random variables to improve the diversity of the population and to bias the search direction. Additionally, a dynamic nonlinear increased crossover probability is utilized to balance the global exploration and local exploitation. COMDE also includes a modified constraint handling technique based on feasibility and the sum of constraints violations. A new dynamic tolerance technique to handle equality constraints is also adopted. However, the test problem contains many equality constraints, considered as a very difficult problem. Thus, in order to increase the number of infeasible solutions to be improved through generations and become feasible with true feasible region, the initial tolerance $a=100$, where $F_{\text {initial }}=-\log _{10}(a), F_{\text {final }}=4, R=0.75$, and the factor equation decreases linearly with $k=1$. The required 


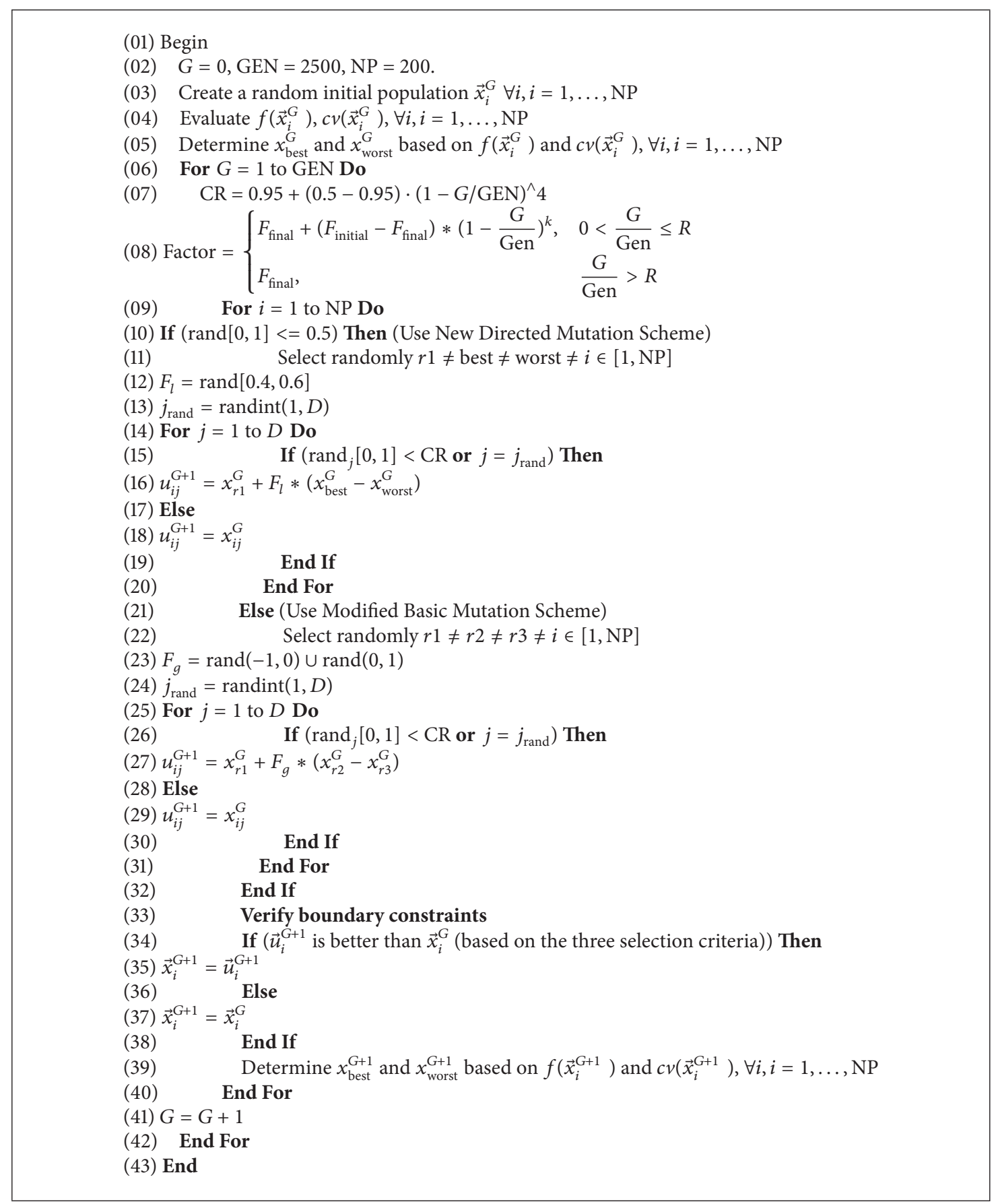

Algorithm 2: Description of COMDE algorithm.

population size NP is 200 and max generation GEN $=2500$. Readers are referred to [26] for details of the designed DE algorithm and its comparative results on benchmark test problems. The working procedure of the designed COMDE algorithm is presented in Algorithm 2.

6.2.1. Handling of Integer Variables. In its canonical form, the differential evolution algorithm and COMDE algorithm are only capable of optimizing unconstrained problems with continuous variables. However, there are very few attempts to transform the canonical DE and proposed COMDE algorithms to handle integer variables [34-37]. In this research, only a couple of simple modifications are required. The new generation of initial population and boundary constraints verification, the proposed novel mutation operation, and the basic mutation schemes use rounding operator, where the operator round $(x)$ rounds the elements of $X$ to the nearest integers. Therefore, the initialization and mutations are as follows: 


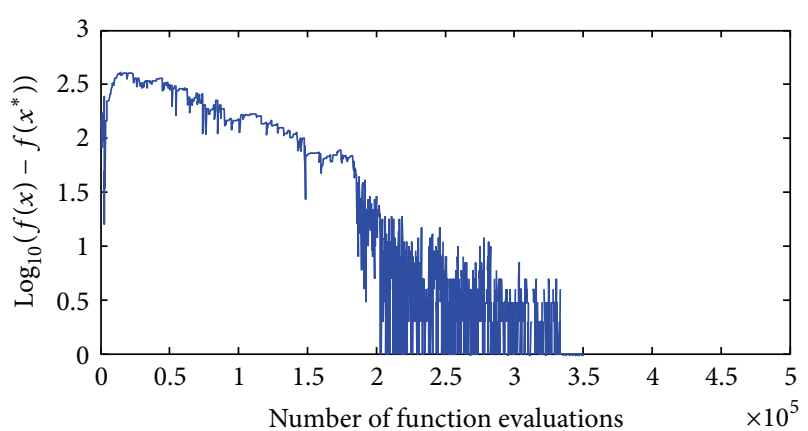

FIGURE 1: Convergence graph (median curve) of COMDE on the test problem of scenario 1 .

(i) Initialization and boundary constraint verification: $x_{i j}^{0}=\operatorname{round}\left(l_{j}+\operatorname{rand}_{j} *\left(u_{j}-l_{j}\right)\right)$.

(ii) New directed mutation: $u_{i j}^{G+1}=\operatorname{round}\left(x_{r 1}^{G}+F_{l} *\left(x_{\text {best }}^{G}{ }^{-}\right.\right.$ $\left.x_{\text {worst }}^{G}\right)$ ).

(iii) Basic mutation: $u_{i j}^{G+1}=\operatorname{round}\left(x_{r 1}^{G}+F_{g} *\left(x_{r 2}^{G}-x_{r 3}^{G}\right)\right)$.

6.2.2. Problem Solution. The proposed GPM for the admission problem discussed in the previous sections has been tested on King Abdulaziz University, and the test results for four scenarios are reported in this section. The experiments were carried out on an Intel Pentium Core 2 due processor $2200 \mathrm{MHZ}$ and 2 GB-RAM. COMDE algorithm is coded and realized in MATLAB. The best result for each scenario in terms of the objective function value and the optimal decision variables is given in Table 2. For each scenario, 30 independent runs are performed and statistical results are provided including the best, median, mean, and worst results and the standard deviation is presented in Table 3 . The convergence graph corresponds to the absolute difference between the best-of-the-run value $f(x)$ and the best optimum solution $f\left(x^{*}\right)$ of the median run of scenario 1 against Total Number of Function Evolutions (TNFE) of the COMDE which is shown in Figure 1.

From Table 2, it can be obviously seen that the best results obtained by COMDE are the optimal solutions for the four scenarios as the constraints are satisfied and the objective functions have the best possible values. Besides, from Table 3, COMDE is unable to reach the best solution consistently in all runs as the problem is very difficult as discussed previously. However, the best and median results are equal and the worst solutions obtained by COMDE are not far from the best with small standard deviations which prove that COMDE is robust technique. Moreover, convergence behavior is another important factor that must be considered in solving optimization problems using evolutionary algorithms. From Figure 1, it can be deduced that the optimal solution can be reached using around $70 \%$ of total number of function evaluations which shows that COMDE is an efficient algorithm with rapid convergence speed. Based on the above results and analysis, it can be concluded that COMDE algorithm has a satisfactory ability to solve considered nonlinear integer GP problem with
TABLE 3: The statistical results of COMDE on the four scenarios.

\begin{tabular}{lccccc}
\hline & Best & Median & Mean & Worst & Std. \\
\hline Scenario 1 & 21,281 & 21281 & 21283.5 & 21310 & 8.93086 \\
Scenario 2 & 22,744 & 22744 & 22748 & 22776 & 10.98170 \\
Scenario 3 & 22,744 & 22744 & 22748 & 22776 & 10.98170 \\
Scenario 4 & 13,516 & 13516 & 13518.3 & 13541 & 11.03416 \\
\hline
\end{tabular}

a good performance in terms of high quality solution and robustness.

\section{Conclusions and Points for Future Researches}

A nonlinear GP model is formulated to satisfy the main objectives stated in the five-year development plan of Saudi Arabia and the 25-year future plan for universities education in the Kingdom. The model is formulated in a general form suitable to be applied in any university in the whole Kingdom; moreover, it can be adopted to suit other goals and/or objectives stated in other countries. Different scenarios revealing various possible priorities of the stated goals for the decision maker are also discussed. An enhanced differential evolution algorithm is used to find the optimum solution for many scenarios representing different priorities for the problem goals. As future researches, it is proposed to consider the following points:

(i) To consider the available jobs in the Saudi market as an additional goal in the proposed model.

(ii) To apply the same model for different universities in Saudi Arabia and for the higher education along the whole Kingdom.

(iii) To formulate a multiobjective mathematical programming model for the same problem.

(iv) To design a complete decision support system with user-friendly input-output interfaces to facilitate the task for the decision makers.

\section{Conflict of Interests}

The authors declare that there is no conflict of interests regarding the publication of this paper.

\section{Acknowledgments}

This project was funded by the National Plan for Science, Technology and Innovation (MAARIFAH), King Abdulaziz City for Science and Technology, Kingdom of Saudi Arabia, award no. 12-INF2234-03. The authors also acknowledge with thanks Science and Technology Unit, King Abdulaziz University, for technical support.

\section{References}

[1] Ministry of Higher Education, Higher Education Report, 2009, https://he.moe.gov.sa/en/Ministry/Deputy-Ministry-for- 
Educational-Affairs/Documents/Higher-Ed-Report-June-09en.pdf.

[2] R. B. Wilkinson, Strategic Enrollment Management: Creating a Definition that Works, Director, Analysis, Planning, \& Assessment, Pittsburg State University, 2007, http://www.educationalpolicy.org/pdf/SEM\%20Guide.pdf.

[3] University of the Fraser Valley Strategic Enrolment Management (SEM) Plan 2014-2019, 2014, http://www.ufv.ca/media/ assets/senate/SEM-2014-Plan-FINAL-Approved-by-theBoard-Sept-4,-2014.pdf.

[4] S. Mansmann and M. H. Scholl, "Decision support system for managing educational capacity utilization," IEEE Transactions on Education, vol. 50, no. 2, pp. 143-150, 2007.

[5] S. F. M. Dahlan and N. A. Yahaya, "A system dynamics model for determining educational capacity of higher education institutions," in Proceedings of the 2nd International Conference on Computational Intelligence, Modelling and Simulation (CIMSim '10), pp. 285-290, September 2010.

[6] E. Tovar and N. Piedra, "Guest editorial: open educational resources in engineering education: various perspectives opening the education of engineers," IEEE Transactions on Education, vol. 57, no. 4, pp. 213-219, 2014.

[7] LINGO, Lingo Optimization Modeling Software for Linear, Nonlinear, and Integer Programming, LINGO, 2009, http://www .lindo.com/.

[8] X.-S. Yang, Metaheristics Optimization: Algorithm Analysis and Open Problems, National Physical Laboratory, Teddington, UK, 2011, http://www.rebennack.net/SEA2011/files/talks/ SEA2011_Yang.pdf.

[9] M. Bashiri, A. P. Manesh, and H. Hasanzadeh, "A heuristic algorithm for nonlinear lexicography goal programming with an efficient initial solution," Journal of Optimization in Industrial Engineering, vol. 15, pp. 77-83, 2014.

[10] S. M. Lee and E. R. Clayton, "A goal programming model for academic resource allocation," Management Science, vol. 18, no. 8, pp. B-395-B-408, 1972.

[11] S. M. Lee and L. J. Moore, "Optimizing university admissions planning," Decision Sciences, vol. 5, no. 3, pp. 405-414, 1974.

[12] K. E. Kendall and R. L. Luebbe, "Management of college student recruiting activities using goal programming," Decision Sciences, vol. 12, no. 2, pp. 193-205, 1981.

[13] H. Khan, "A product mix model of linear programming for University's optimal enrollment management," in Proceedings of the Conference for Industry and Education Collaboration, American Society for Engineering Education, Orlando, Fla, USA, 2009.

[14] E. Gottlieb, "Using integer programming to guide college admissions decisions: a preliminary report, consortium for computing in small colleges," Journal of Computing Sciences in Colleges, vol. 17, no. 2, pp. 271-279, 2001.

[15] P. K. Sugrue, "An optimization model for the allocation of university based merit aid," Journal of Student Financial Aid, vol. 40, no. 2, pp. 21-30, 2010.

[16] A. S. Mashat, A. M. Ragab, and A. M. Khedra, "Decision support system based Markov model for performance evaluation of students flow in FCIT-KAU," in Proceedings of the ICCIT, pp. 409-414, 2012.

[17] B. A. Kassa, "A linear programming approach for placement of applicants to academic programs," SpringerPlus, vol. 2, no. 1, pp. $1-7,2013$.
[18] A. H. Ragab, A. Y. Noaman, A. S. Al-Ghamdi, and A. I. Madbouly, "A comparative analysis of classification algorithms for students college enrollment approval using data mining," in Proceedings of the Workshop on Interaction Design in Educational Environments (IDEE '14), pp. 106-113, Albacete, Spain, June 2014.

[19] A. M. Ragab, A. S. Mashat, and A. M. Khedra, "Design and implementation of a hybrid recommender system for predicting college admission," International Journal of Computer Information Systems and Industrial Management Applications, vol. 6, pp. 35-44, 2014.

[20] Ministry of Economy and Planning, Ninth National Development Plan, Ministry of Economy and Planning, Riyadh, Saudi Arabia, 2014, http://www.saudi.gov.sa.

[21] Ministry of Higher Education, The Future Plan for University Education in the Kingdom of Saudi Arabia, (AAFAQ), Al Riyadh, Saudi Arabia, 2014, http://aafaq.mohe.gov.sa/default.aspx.

[22] Information and Statistics Department, Statistical Report, Deanship of Information Technology, King Abdulaziz University, 2014.

[23] Ministry of Higher Education, Kingdom of Saudi Arabia, 2014, http://www.mohe.gov.sa.

[24] Central Department of Statistics and Information, Statistical Year Book, Central Department of Statistics and Information, Riyadh, Saudi Arabia, 2013.

[25] Central Department of Statistics and Information (Saudi Arabia), Statistical Year Book, 2014.

[26] A. W. Mohamed and H. Z. Sabry, "Constrained optimization based on modified differential evolution algorithm," Information Sciences, vol. 194, pp. 171-208, 2012.

[27] S. Das and P. N. Suganthan, "Differential evolution: a survey of the state-of-the-art," IEEE Transactions on Evolutionary Computation, vol. 15, no. 1, pp. 4-31, 2011.

[28] R. Storn and K. Price, "Differential evolution-a simple and efficient adaptive scheme for global optimization over continuous spaces," Tech. Rep. TR-95-012, ICSI, 1995.

[29] K. V. Price, R. M. Storn, and J. A. Lampinen, Differential Evolution: A Practical Approach to Global Optimization, Springer, Berlin, Germany, 2005.

[30] R. Storn and K. Price, "Differential evolution-a simple and efficient heuristic for global optimization over continuous spaces," Journal of Global Optimization, vol. 11, no. 4, pp. 341359, 1997.

[31] A. W. Mohamed, H. Z. Sabry, and A. Farhat, "Advanced differential Evolution algorithm for global numerical optimizatiom," in Proceedings of the IEEE International Conference on Computer Applications and Industrial Electronics (ICCAIE '11), pp. 156-161, IEEE, Penang, Malaysia, December 2011.

[32] A. W. Mohamed, "RDEL: restart differential evolution algorithm with local search mutation for global numerical optimization," Egyptian Informatics Journal, vol. 15, no. 3, pp. 175-188, 2014.

[33] A. W. Mohamed, H. Z. Sabry, and T. Abd-Elaziz, "Real parameter optimization by an effective differential evolution algorithm," Egyptian Informatics Journal, vol. 14, no. 1, pp. 37-53, 2013.

[34] J. Lampinen and I. Zelinka, "Mixed integer-discrete-continuous optimization by differential evolution, part 1: the optimization method," in Proceedings of the 5th International Mendel Conference on Soft Computing (MENDEL '99), P. Ošmera, Ed., Brno, Czech Republic, June 1999. 
[35] J. Gao, H. Li, and Y.-C. Jiao, "Modified differential evolution for the integer programming problems," in Proceedings of the International Conference on Artificial Intelligence and Computational Intelligence (AICI '09), vol. 1, pp. 213-219, IEEE, Shanghai, China, November 2009.

[36] M. G. H. Omran and A. P. Engelbrecht, "Differential evolution for integer programming problems," in Proceedings of the IEEE Congress on Evolutionary Computation (CEC '07), pp. 22372242, September 2007.

[37] W. Jun, Y. Gao, and Y. Lina, "An improved differential evolution algorithm for mixed integer programming problems," in Proceedings of the 9th International Conference on Computational Intelligence and Security (CIS '13), pp. 31-35, December 2013. 


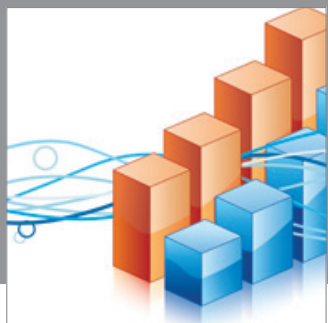

Advances in

Operations Research

mansans

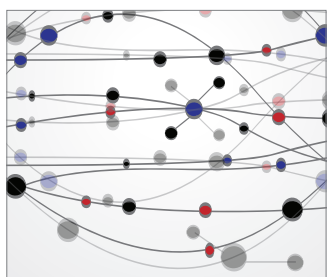

The Scientific World Journal
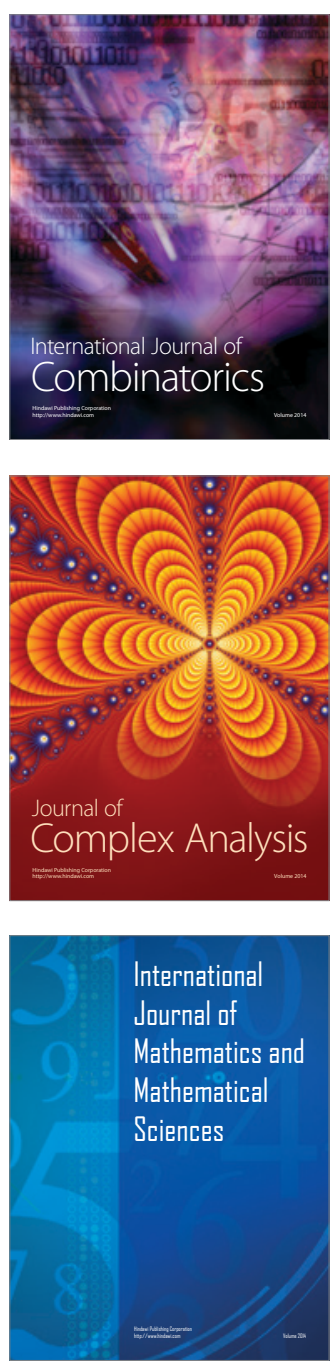
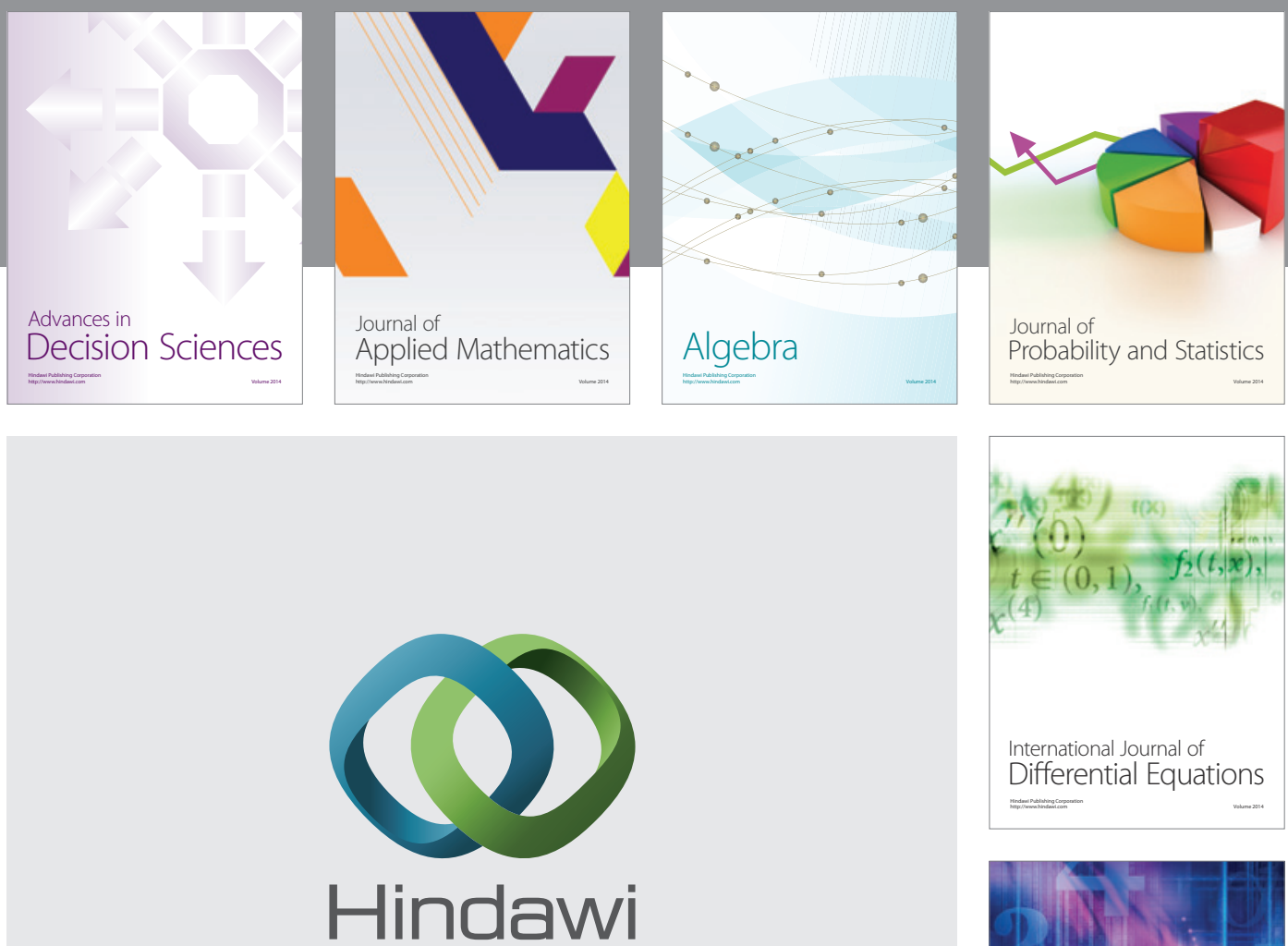

Submit your manuscripts at http://www.hindawi.com
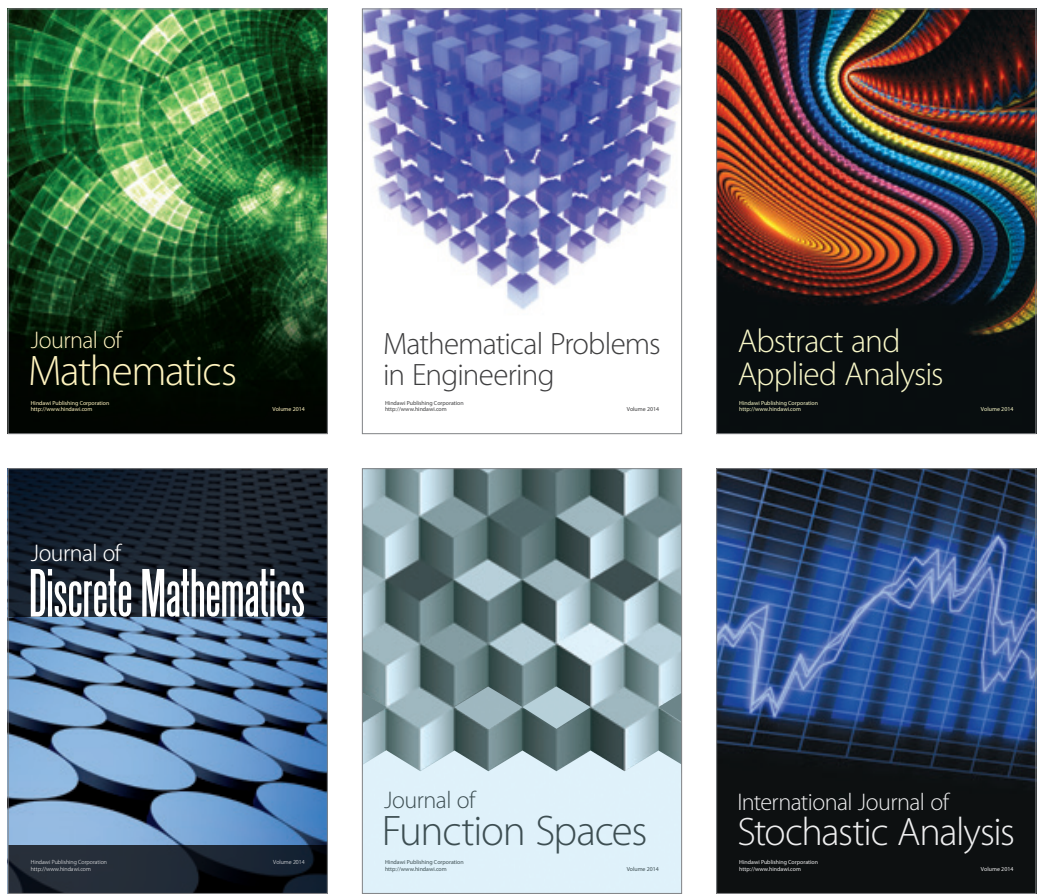

Journal of

Function Spaces

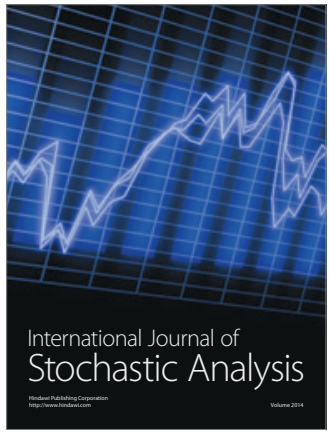

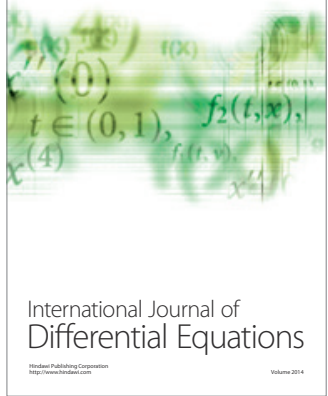
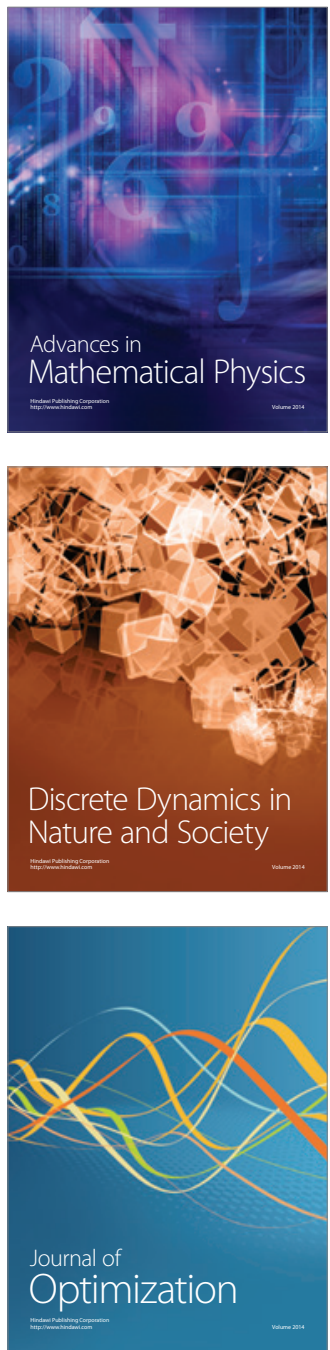